Pacific

Journal of

Mathematics

HIGHER HOMOTOPY COMMUTATIVITY OF $H$-SPACES WITH FINITELY GENERATED COHOMOLOGY

Yusuke KaWAMOTO

Volume $204 \quad$ No. 1

May 2002 


\title{
HIGHER HOMOTOPY COMMUTATIVITY OF H-SPACES WITH FINITELY GENERATED COHOMOLOGY
}

\author{
Yusuke KaWAMOTO
}

\begin{abstract}
Suppose $X$ is a simply connected $\bmod p H$-space such that the $\bmod p$ cohomology $H^{*}(X ; \mathbb{Z} / p)$ is finitely generated as an algebra. Our first result shows that if $X$ is an $A_{n}$-space, then $X$ is the total space of a principal $A_{n}$-fibration with base a finite $A_{n}$-space and fiber a finite product of $\mathbb{C} P^{\infty}$ s. As an application of the first result, it is shown that if $X$ is a quasi $C_{p^{-}}$-space, then $X$ is homotopy equivalent to a finite product of $\mathbb{C P}{ }^{\infty} \mathrm{s}$.
\end{abstract}

\section{Introduction.}

The theory of $\mathrm{H}$-spaces is a generalization of the homotopy theory of Lie groups, and it has been investigated as one of the most important objects of study in algebraic topology. It is useful to consider the $H$-spaces at a prime by using the completion of Bousfield-Kan [2]. Given a prime $p$, an $H$-space which is completed at $p$ is called a $\bmod p H$-space. In this paper, homotopy equivalence means $\bmod p$ homotopy equivalence and cohomology is $\bmod p$ cohomology unless otherwise specified.

In recent decades, many theorems have been proved about mod $p$ finite $H$-spaces (cf. [6], [12] and [17]), which suggest that they are similar to Lie groups. In this paper, we study $\bmod p H$-spaces which need not be finite, but whose cohomology rings are finitely generated. It is known that the threeconnected cover $G\langle 3\rangle$ of a Lie group $G$ is such an $H$-space. Another example is the infinite dimensional complex projective space $\mathbb{C} P^{\infty}$. Recently, Broto and Crespo [3] and [4] have obtained remarkable results about mod $p H$ spaces with finitely generated cohomology. It follows from their results that such an $H$-space is the total space of a principal $H$-fibration with base a $\bmod p$ finite $H$-space and fiber a product of $\mathbb{C} P^{\infty}$ s. One of the purposes of this paper is to generalize their results to the case of higher homotopy associative mod $p H$-spaces with finitely generated cohomology.

Stasheff [22] introduced the notion of the higher homotopy associativity of $H$-spaces as a series of intermediate stages between $H$-spaces and loop spaces. An $A_{2}$-space is an $H$-space with a multiplication $M_{2}: X \times X \rightarrow$ $X$, and an $A_{3}$-space is a homotopy associative $H$-space. Now we denote $M_{2}(x, y)=x y$ for $x, y \in X$. Let $X$ be an $A_{3}$-space, and let $M_{3}: I \times X^{3} \rightarrow X$ 
be a map satisfying that $M_{3}(0, x, y, z)=(x y) z$ and $M_{3}(1, x, y, z)=x(y z)$ for $x, y, z \in X$. By using the map $M_{3}$, we can define a map $\widetilde{M}_{4}: S^{1} \times X^{4} \rightarrow X$ such that $\widetilde{M}_{4}(t, x, y, z, w)$ is the pentagon in Figure 1 .

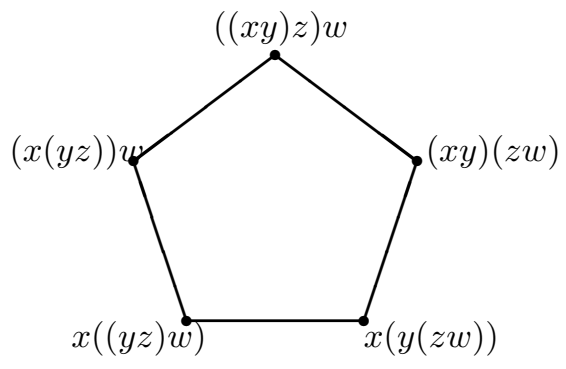

Figure 1. $A_{4}$-form on $X$.

$X$ is said to be an $A_{4}$-space if there exists a map $M_{4}: D^{2} \times X^{4} \rightarrow X$ with $\left.M_{4}\right|_{S^{1} \times X^{4}}=\widetilde{M}_{4}$. In general, an $H$-space $X$ is called an $A_{n}$-space if there exists an $A_{n}$-form $\left\{M_{i}: D^{i-2} \times X^{i} \rightarrow X\right\}_{2 \leq i \leq n}$ satisfying some conditions. Figure 2 denotes the $A_{5}$-form on $X$ (see $\S 2$ ). Furthermore, an $A_{\infty}$-space has the homotopy type of a loop space. Similarly, an $A_{n}$-map is defined as an $H$-map between $A_{n}$-spaces preserving the $A_{n}$-forms (see $\S 2$ ). An $H$-fibration consisting of $A_{n}$-spaces and $A_{n}$-maps is called an $A_{n}$-fibration.

Our first result is stated as follows:

Theorem A. If $X$ is a simply connected $A_{n}$-space such that the mod $p$ cohomology $H^{*}(X ; \mathbb{Z} / p)$ is finitely generated as an algebra, then we have a simply connected finite $A_{n}$-space $Y$ and a principal $A_{n}$-fibration

$$
F \longrightarrow X \longrightarrow Y \text {, }
$$

where the fiber $F$ is the direct product of a finite number of $\mathbb{C} P^{\infty}$ s.

The above theorem is regarded as a generalization of [3, Thm. 1.1] and [4, Thm. 1.1] since $H$-space is the same as $A_{2}$-space. From Theorem A, it is possible to reduce a problem about $A_{n}$-spaces with finitely generated cohomology to the case of finite $A_{n}$-spaces. In this paper, Theorem $\mathrm{A}$ is used to study the higher homotopy commutativity of $H$-spaces with finitely generated cohomology. In the case of $p=2$, Slack has shown the following result:

Theorem 1.1 ([21, Thm. 0.1]). If $X$ is a simply connected homotopy commutative mod $2 H$-space such that the $\bmod 2$ cohomology $H^{*}(X ; \mathbb{Z} / 2)$ is finitely generated as an algebra, then $X$ is homotopy equivalent to a finite product of $\mathbb{C} P^{\infty} s$.

Here we note that Broto-Crespo [3, Cor. 1.5] gave another proof of Theorem 1.1. On the other hand, the odd prime version of Theorem 1.1 does 
not hold. In fact, it was shown by Iriye-Kono [9, Thm. 1.3] that for an odd prime $p$, any connected mod $p H$-space possesses a multiplication which is homotopy commutative. Furthermore, one may expect that a simply connected homotopy commutative mod $p$ loop space with finitely generated cohomology has the homotopy type of a product of $\mathbb{C} P^{\infty}$ s. However, we see that by McGibbon [18, Thm. 2], $S p(2)$ for $p=3$ and $S^{3}$ for $p \geq 5$ are counterexamples.

To describe an odd prime version of Theorem 1.1, we need to generalize the homotopy commutativity of $H$-spaces to the higher ones. Such notions were first considered by Sugawara [24] and Williams [25] in the case of loop spaces. Later Hemmi [8] introduced the higher homotopy commutativity of $H$-spaces. Let $X$ be an $A_{n}$-space, and let $P_{i}(X)$ denote the $i$-th projective space of $X$ for $1 \leq i \leq n$. A quasi $C_{n}$-form on $X$ is defined by a system of maps $\left\{\lambda_{i}:(\Sigma X)^{i} \rightarrow P_{i}(X)\right\}_{1 \leq i \leq n}$ satisfying some conditions (see $\S 3$ ). An $A_{n}$-space which has a quasi $C_{n}$-form is called a quasi $C_{n}$-space. In $[8, \mathrm{Thm}$. 1.1], Hemmi has shown that if $X$ is a simply connected finite quasi $C_{p}$-space, then $X$ is contractible (see Theorem 3.3). Now we generalize his result to the case of quasi $C_{p}$-spaces with finitely generated cohomology.

Theorem B. If $X$ is a simply connected quasi $C_{p}$-space such that the $\bmod p$ cohomology $H^{*}(X ; \mathbb{Z} / p)$ is finitely generated as an algebra, then $X$ is homotopy equivalent to a finite product of $\mathbb{C} P^{\infty} s$.

Theorem B was first conjectured by Slack [21, pp. 4-5], and was suggested to the author by Lin. In the above theorem, it is impossible to relax the condition of quasi $C_{p}$-space to quasi $C_{p-1}$-space. In fact, by [8, Thm. 2.4], the odd dimensional sphere $S^{2 n-1}$ is a quasi $C_{p-1}$-space for any $n \geq 1$. Now we note that Theorem B implies Theorem 1.1 since a homotopy commutative $H$-space is a quasi $C_{2}$-space by [8, Prop. 2.3] (see also [23, Thm. 13.6]). Furthermore, in the case that $X$ is a loop space, by [8, Thm. 2.2], quasi $C_{n}$-space is the same condition as $C_{n}$-space in the sense of Williams $[\mathbf{2 5}$, Def. 5] (see also [20, Thm. 3.2]), and so Theorem B implies [14, Thm. C]. In particular, since the loop space of an $H$-space is a quasi $C_{n}$-space for all $n \geq 1$, we have the following result (see also $[\mathbf{1 5}]$ and $[\mathbf{1 6}]$ ):

Theorem $1.2([\mathbf{1 3}$, Thm. A]). Let $X$ be a simply connected $\bmod p H$-space with finitely generated $\bmod p$ cohomology. If $X$ has the homotopy type of the loop space of an $H$-space, then $X$ is homotopy equivalent to a finite product of $\mathbb{C} P^{\infty} s$.

This paper is organized as follows: In $\S 2$, we recall the nullification functor and the colocalization functor introduced by Dror Farjoun [5]. It is shown that those homotopy functors preserve the higher homotopy associativity of $H$-spaces (see Theorem 2.1). $\S 3$ is devoted to the proofs of Theorem A and Theorem B. First we recall the results of Broto and Crespo [3] and 
[4] about $H$-spaces with finitely generated cohomology. By combining their results with Theorem 2.1 obtained in $\S 2$, we can prove Theorem A. To prove Theorem B, we show that the nullification functor $L_{B \mathbb{Z} / p}$ with respect to $B \mathbb{Z} / p$ preserves a quasi $C_{n}$-form (see Theorem 3.5). As a consequence of Theorem A and Theorem 3.5, we obtain the proof of Theorem B.

The content of the paper was first presented in JAMI conference on homotopy theory at Johns Hopkins University in March 2000. The author is grateful to the organizers for their kind invitation and hospitality. I would also like to thank Jim Stasheff for many helpful comments on the manuscript of the paper. Furthermore, I wish to express my appreciation to Yutaka Hemmi and Jim Lin for many discussions about the higher homotopy commutativity of $H$-spaces. In particular, Theorem $\mathrm{B}$ was suggested to me by Jim Lin. Finally, I am grateful to Takao Matumoto and Mitsunori Imaoka for their encouragements.

\section{Nullification functor and colocalization functor.}

Dror Farjoun [5] introduced the nullification functor and the colocalization functor with respect to spaces. Let $\mathcal{S}_{*}$ denote the category of pointed spaces having the homotopy types of $C W$-complexes, and let $A \in \mathcal{S}_{*}$. A space $X \in$ $\mathcal{S}_{*}$ is called $A$-null if the pointed mapping space $\operatorname{Map}_{*}(A, X)$ is contractible. By Dror Farjoun [5, Thm. 1.A.3], we have the $A$-nullification functor $L_{A}$ : $\mathcal{S}_{*} \rightarrow \mathcal{S}_{*}$. Given a space $X$, the $A$-nullification $L_{A}(X)$ is $A$-null, and we have the natural map $\phi_{X}: X \rightarrow L_{A}(X)$. By [5, Thm. 1.C.1], $\phi_{X}$ is homotopically universal, that is, for any $A$-null space $Z$ and a map $\zeta: X \rightarrow Z$, there exists a map $\widetilde{\zeta}: L_{A}(X) \rightarrow Z$ unique up to homotopy such that $\widetilde{\zeta} \phi_{X} \simeq \zeta$. Furthermore, it is known by [5, p. 18] that the natural map $\phi_{X}$ induces a homotopy equivalence

$$
\left(\phi_{X}\right)^{*}: \operatorname{Map}_{*}\left(L_{A}(X), Z\right) \longrightarrow \operatorname{Map}_{*}(X, Z)
$$

for an $A$-null space $Z$.

Let $X, Y \in \mathcal{S}_{*}$. A map $f: Y \rightarrow X$ is called an $A$-equivalence if the induced map

$$
f_{*}: \operatorname{Map}_{*}(A, Y) \longrightarrow \operatorname{Map}_{*}(A, X)
$$

is a homotopy equivalence. In [5, Prop. 2.B.1], Dror Farjoun constructed the $A$-colocalization functor $C W_{A}: \mathcal{S}_{*} \rightarrow \mathcal{S}_{*}$. Given a space $X$, we have the natural map $\psi_{X}: C W_{A}(X) \rightarrow X$ which is an $A$-equivalence. By $[\mathbf{5}$, Thm. 2.B.3], the natural map $\psi_{X}$ is homotopically universal among all $A$ equivalences, that is, for any $A$-equivalence $\xi: Y \rightarrow \underset{\widetilde{\xi}}{X}$, there exists a map $\widetilde{\xi}: C W_{A}(X) \rightarrow Y$ unique up to homotopy such that $\xi \widetilde{\xi} \simeq \psi_{X}$. If the natural $A$-equivalence $\psi_{X}: C W_{A}(X) \rightarrow X$ is a homotopy equivalence, then $X$ is called an $A$-cellular space. 
To prove Theorem A, we show that those homotopy functors $L_{A}$ and $C W_{A}$ preserve the higher homotopy associativity of $H$-spaces (see Theorem 2.1). Now we recall the definitions of an $A_{n}$-space and an $A_{n}$-map introduced by Stasheff [22] and Iwase-Mimura [11], respectively.

Stasheff [22] introduced the notion of the higher homotopy associativity of $H$-spaces. To describe an $A_{n}$-form on an $H$-space, he defined a special complex $K_{i}$ which is homeomorphic to the $(i-2)$-dimensional disk for $i \geq 2$. Let $L_{i}=\partial K_{i}$ denote the boundary of the complex $K_{i}$. Then $L_{i}$ is the union of $(i(i-1) / 2-1)$-faces $K_{k}(r, s)$ for $r, s \geq 2,1 \leq k \leq r, r+s=i+1$, and the face $K_{k}(r, s)$ is homeomorphic to $K_{r} \times K_{s}$ by the face operator $\partial_{k}(r, s): K_{r} \times K_{s} \rightarrow K_{k}(r, s)$.

An $A_{n}$-form on $X$ consists of a system of maps $\left\{M_{i}: K_{i} \times X^{i} \rightarrow X\right\}_{2 \leq i \leq n}$ satisfying the following conditions:

$$
M_{2}(x, *)=M_{2}(*, x)=x,
$$

that is, $M_{2}: X^{2} \rightarrow X$ is a multiplication with unit, where $K_{2} \times X^{2}=$ $\{*\} \times X^{2}$ is identified with $X^{2}$.

$$
\begin{aligned}
& M_{i}\left(\partial_{k}(r, s)(\rho, \sigma), x_{1}, \ldots, x_{i}\right) \\
& =M_{r}\left(\rho, x_{1}, \ldots, x_{k-1}, M_{s}\left(\sigma, x_{k}, \ldots, x_{k+s-1}\right), x_{k+s}, \ldots, x_{i}\right)
\end{aligned}
$$

for $(\rho, \sigma) \in K_{r} \times K_{s}$.

$$
\begin{aligned}
& M_{i}\left(\tau, x_{1}, \ldots, x_{j-1}, *, x_{j+1}, \ldots, x_{i}\right) \\
& =M_{i-1}\left(s_{j}(\tau), x_{1}, \ldots, x_{j-1}, x_{j+1}, \ldots, x_{i}\right),
\end{aligned}
$$

where $s_{j}: K_{i} \rightarrow K_{i-1}$ denotes the degeneracy map for $1 \leq j \leq i$ (see [22, I Prop. 3]). A space $X$ together with an $A_{n}$-form is called an $A_{n}$-space. If $X$ has a system of maps $\left\{M_{i}: K_{i} \times X^{i} \rightarrow X\right\}_{i \geq 2}$ such that $\left\{M_{i}\right\}_{2 \leq i \leq n}$ is an $A_{n}$-form on $X$ for any $n \geq 2$, then $X$ is called an $A_{\infty}$-space.

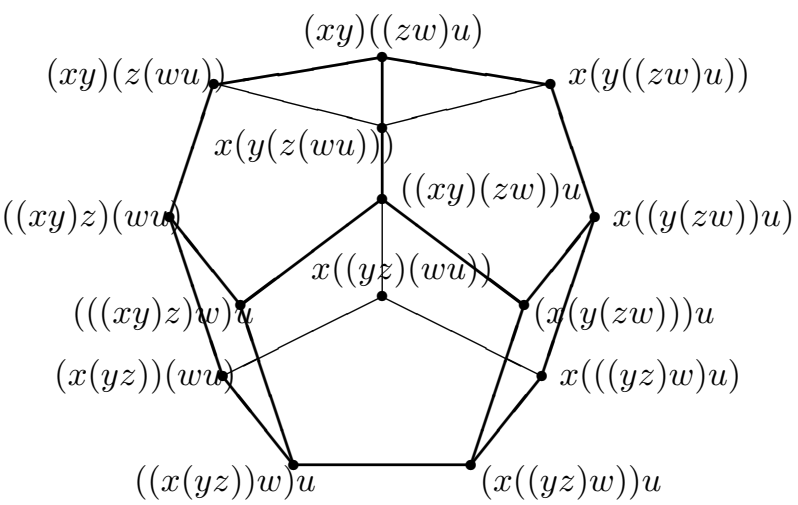

Figure 2. $A_{5}$-form on $X$. 
It is natural to consider the notion of the higher homotopy associativity of maps between $A_{n}$-spaces. Such notions were first considered by Sugawara $[\mathbf{2 4}, \S 2]$ and Stasheff [23, Def. 11.9] under some restricted situations. The full generality was described by Iwase-Mimura [11]. To construct an $A_{n^{-}}$ form of a map, they defined a special complex $\Gamma_{i}$ which is homeomorphic to the $(i-1)$-dimensional disk for $i \geq 1$. Let $\Lambda_{i}=\partial \Gamma_{i}$ denote the boundary of the complex $\Gamma_{i}$. Then $\Lambda_{i}$ is the union of the following $\left(i(i-1) / 2+2^{i-1}-1\right)$ faces:

$$
\begin{array}{ll}
\Gamma_{k}(r, s) & \text { for } 1 \leq k \leq r, 1 \leq r \leq i-1, r+s=i+1, \\
\Gamma\left(t ; r_{1}, \ldots, r_{t}\right) & \text { for } 2 \leq t \leq i, r_{j} \geq 1, r_{1}+\cdots+r_{t}=i,
\end{array}
$$

and the faces $\Gamma_{k}(r, s)$ and $\Gamma\left(t ; r_{1}, \ldots, r_{t}\right)$ are homeomorphic to $\Gamma_{r} \times K_{s}$ and $K_{t} \times \Gamma_{r_{1}} \times \cdots \times \Gamma_{r_{t}}$, respectively. The homeomorphisms $\delta_{k}(r, s): \Gamma_{r} \times K_{s} \rightarrow$ $\Gamma_{k}(r, s)$ and $\delta\left(t ; r_{1}, \ldots, r_{t}\right): K_{t} \times \Gamma_{r_{1}} \times \cdots \times \Gamma_{r_{t}} \rightarrow \Gamma\left(t ; r_{1}, \ldots, r_{t}\right)$ are called the face operators on $\Gamma_{i}$. Furthermore, we have the degeneracy operations $d_{j}: \Gamma_{i} \rightarrow \Gamma_{i-1}$ for $1 \leq j \leq i$ satisfying certain conditions (see [11, (2-d)]).

From the construction of $\Gamma_{i}$, there exists a homeomorphism $\zeta_{i}: I \times K_{i} \rightarrow$ $\Gamma_{i}$ such that $\zeta_{i}\left(\{1\} \times K_{i}\right)=\Gamma_{1}(1, i), \zeta_{i}\left(\{0\} \times K_{i}\right)=\Gamma(i ; 1, \ldots, 1)$ and

$$
\zeta_{i}\left(I \times L_{i}\right)=\bigcup_{(k, r, s) \in \Phi_{i}} \Gamma_{k}(r, s) \cup \bigcup_{\left(t ; r_{1}, \ldots, r_{t}\right) \in \Psi_{i}} \Gamma\left(t ; r_{1}, \ldots, r_{t}\right),
$$

where $\Phi_{i}=\{(k, r, s) \mid 1 \leq k \leq r, 2 \leq r \leq i-1, r+s=i+1\}$ and $\Psi_{i}=\left\{\left(t ; r_{1}, \ldots, r_{t}\right) \mid 2 \leq t \leq i-1, r_{j} \geq 1, r_{1}+\cdots+r_{t}=i\right\}$. By using the homeomorphism $\zeta_{i}$, we identify the complex $\Gamma_{i}$ with $I \times K_{i}$.

Let $X$ and $Y$ be $A_{n}$-spaces, and let $\phi: X \rightarrow Y$ be a map. Then we have the $A_{n}$-forms $\left\{M_{i}: K_{i} \times X^{i} \rightarrow X\right\}_{2 \leq i \leq n}$ and $\left\{N_{i}: K_{i} \times Y^{i} \rightarrow Y\right\}_{2 \leq i \leq n}$ on $X$ and $Y$, respectively. An $A_{n}$-form on the map $\phi: X \rightarrow Y$ is a system of maps $\left\{F_{i}: \Gamma_{i} \times X^{i} \rightarrow Y\right\}_{1 \leq i \leq n}$ satisfying the following conditions:

$$
F_{1}=\phi: X \longrightarrow Y,
$$

where $\Gamma_{1} \times X=\{*\} \times X$ is identified with $X$.

$$
\begin{aligned}
& F_{i}\left(\delta_{k}(r, s)(\rho, \sigma), x_{1}, \ldots, x_{i}\right) \\
& \quad=F_{r}\left(\rho, x_{1}, \ldots, x_{k-1}, M_{s}\left(\sigma, x_{k}, \ldots, x_{k+s-1}\right), x_{k+s}, \ldots, x_{i}\right)
\end{aligned}
$$

for $(\rho, \sigma) \in \Gamma_{r} \times K_{s}$.

$$
\begin{aligned}
& F_{i}\left(\delta\left(t ; r_{1}, \ldots, r_{t}\right)\left(\tau, \rho_{1}, \ldots, \rho_{t}\right), x_{1}, \ldots, x_{i}\right) \\
& \quad=N_{t}\left(\tau, F_{r_{1}}\left(\rho_{1}, x_{1}, \ldots, x_{r_{1}}\right), \ldots, F_{r_{t}}\left(x_{r_{1}+\cdots+r_{t-1}+1}, \ldots, x_{i}\right)\right)
\end{aligned}
$$

for $\left(\tau, \rho_{1}, \ldots, \rho_{t}\right) \in K_{t} \times \Gamma_{r_{1}} \times \cdots \times \Gamma_{r_{t}}$.

$$
\begin{aligned}
& F_{i}\left(\gamma, x_{1}, \ldots, x_{j-1}, *, x_{j+1}, \ldots, x_{i}\right) \\
& \quad=F_{i-1}\left(d_{j}(\gamma), x_{1}, \ldots, x_{j-1}, x_{j+1}, \ldots, x_{i}\right)
\end{aligned}
$$

for $1 \leq j \leq i$. 


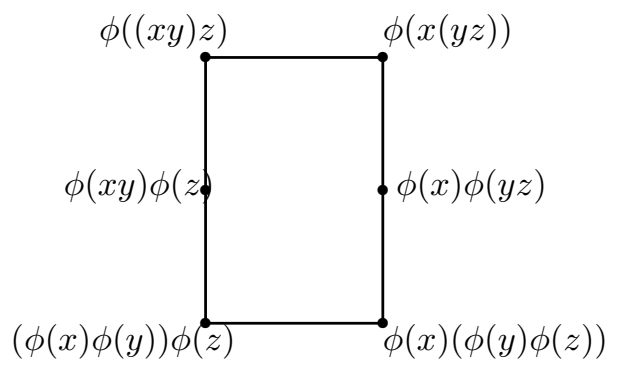

Figure 3. $A_{3}$-form on $\phi$.

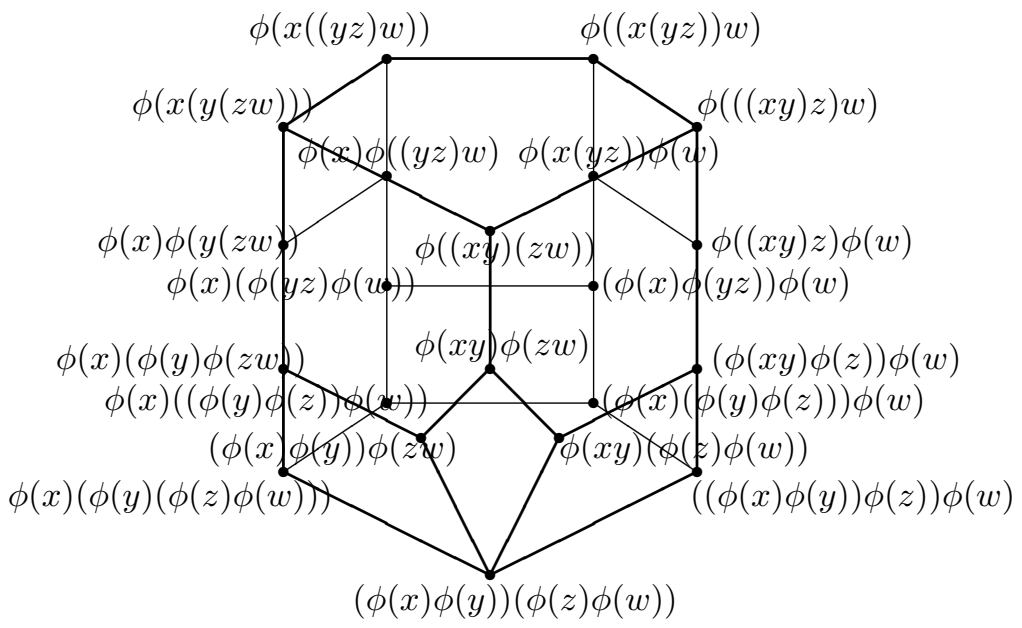

Figure 4. $A_{4}$-form on $\phi$.

Figure 3 and Figure 4 denote the $A_{3}$-form and the $A_{4}$-form on $\phi$, respectively. A map together with an $A_{n}$-form is called an $A_{n}$-map. From the definition, we see that an $A_{2}$-map and an $A_{3}$-map are an $H$-map and an $H$-map preserving the homotopy associativity of $H$-spaces, respectively. Furthermore, an $A_{\infty}$-map is homotopic to a loop map, and we have the induced map between classifying spaces (see [12, §6.4]).

In the proof of Theorem A, we need the following result:

Theorem 2.1. Let $A, X \in \mathcal{S}_{*}$. Then we have the following:

(1) If $X$ is an $A_{n}$-space, then the A-nullification $L_{A}(X)$ is an $A_{n}$-space and the natural map $\phi_{X}: X \rightarrow L_{A}(X)$ is an $A_{n}$-map.

(2) If $X$ is an $A_{n}$-space, then the $A$-colocalization $C W_{A}(X)$ is an $A_{n}$-space and the natural map $\psi_{X}: C W_{A}(X) \rightarrow X$ is an $A_{n}$-map. 
A functor $F: \mathcal{S}_{*} \rightarrow \mathcal{S}_{*}$ is called continuous if the map

$$
\lambda_{F}: \operatorname{Map}_{*}(X, Y) \longrightarrow \operatorname{Map}_{*}(F(X), F(Y))
$$

defined by $\lambda_{F}(g)=F(g)$ is continuous when the compact-open topology are assigned to those mapping spaces. Let $(\mathbb{Z} / p)_{\infty}$ denote the $p$-completion functor of Bousfield-Kan [2]. It is shown by Iwase [10, Thm. 3.7] that $(\mathbb{Z} / p)_{\infty}$ is a continuous functor. He used the result to show that $(\mathbb{Z} / p)_{\infty}$ strictly preserves the higher homotopy associativity of $H$-spaces (see $[\mathbf{1 0}$, Cor. 3.10]). Since the continuity of $L_{A}$ and $C W_{A}$ are proved by Dror Farjoun $[\mathbf{5}$, Thm. 1.A.3, Thm. 2.B.3], we also use a similar way to the proof of $[\mathbf{1 0}$, Thm. 3.7, Cor. 3.10] to prove Theorem 2.1. But we need to argue more precisely than [10] since $L_{A}$ and $C W_{A}$ are in general homotopy functors. Theorem 2.1 shows that $L_{A}$ and $C W_{A}$ preserve the higher homotopy associativity of $H$-spaces up to homotopy.

Kawamoto [14, Thm. 2.14] has shown that the nullification functor $L_{A}$ preserves the higher homotopy commutativity in the sense of Williams [25] of loop spaces. Since the proof of Theorem 2.1(1) is similar to the one of [14, Thm. 2.14], we give an outline of the proof.

Proof of Theorem 2.1(1). Since $X$ is an $A_{n}$-space, there is a system of maps $\left\{M_{i}: K_{i} \times X^{i} \rightarrow X\right\}_{2 \leq i \leq n}$ satisfying Conditions (2.2)-(2.4). By using induction on $i$, we construct $A_{n}$-forms $\left\{N_{i}: K_{i} \times L_{A}(X)^{i} \rightarrow L_{A}(X)\right\}_{2 \leq i \leq n}$ and $\left\{F_{i}: \Gamma_{i} \times X^{i} \rightarrow L_{A}(X)\right\}_{1 \leq i \leq n}$ on $L_{A}(X)$ and $\phi_{X}$ satisfying Conditions (2.2)-(2.4) and (2.5)-(2.8), which implies the required conclusion.

First we put $F_{1}=\phi_{X}: X \rightarrow L_{A}(X)$, where $\Gamma_{1} \times X$ is identified with $X$. Next we identify $K_{2} \times L_{A}(X)^{2}$ and $\Gamma_{2} \times X^{2}$ with $L_{A}(X)^{2}$ and $I \times$ $X^{2}$, respectively. By $[5,1 . A .8$ e.4], there is a homotopy equivalence $\gamma$ : $L_{A}(X)^{2} \rightarrow L_{A}\left(X^{2}\right)$ with $\gamma\left(\phi_{X}\right)^{2} \simeq \phi_{X^{2}}$. If we define a map $\widetilde{N}_{2}: L_{A}(X)^{2} \rightarrow$ $L_{A}(X)$ by the composite $\widetilde{N}_{2}=L_{A}\left(M_{2}\right) \gamma$, then $\widetilde{N}_{2}\left(\phi_{X}\right)^{2} \simeq \phi_{X} M_{2}$. Since $\tilde{N}_{2} \iota_{j} \phi_{X} \simeq \phi_{X}$, we have by $(2.1)$ that $\widetilde{N}_{2} \iota_{j} \simeq 1_{L_{A}(X)}$ for $j=1,2$, where $\iota_{j}: L_{A}(X) \rightarrow L_{A}(X)^{2}$ denotes the inclusion on the $j$-th factor. From the homotopy extension property, there is a map $N_{2}: L_{A}(X)^{2} \rightarrow L_{A}(X)$ with $N_{2} \iota_{j}=1_{L_{A}(X)}$ for $j=1,2$ and $N_{2}\left(\phi_{X}\right)^{2} \simeq \phi_{X} M_{2}$. Here we can choose a homotopy $F_{2}: I \times X^{2} \rightarrow L_{A}(X)$ such that $F_{2}(t, x, *)=F_{2}(t, *, x)=\phi_{X}(x)$, $\left.F_{2}\right|_{\{0\} \times X^{2}}=N_{2}\left(\phi_{X}\right)^{2}$ and $\left.F_{2}\right|_{\{1\} \times X^{2}}=\phi_{X} M_{2}$.

By the inductive hypothesis, we have systems of maps $\left\{N_{j}: K_{j} \times L_{A}(X)^{j}\right.$ $\rightarrow Y\}_{2 \leq j \leq i-1}$ and $\left\{F_{j}: \Gamma_{j} \times X^{j} \rightarrow L_{A}(X)\right\}_{1 \leq j \leq i-1}$ satisfying Conditions (2.2)-(2.4) and (2.5)-(2.8). Now we put that $S_{i}=I \times\left(L_{i} \times X^{i} \cup K_{i} \times X^{[i]}\right) \cup$ $\{1\} \times K_{i} \times X^{i}$ and $T_{i}=\left(\zeta_{i} \times 1_{X^{i}}\right)\left(S_{i}\right) \subset \Gamma_{i} \times X^{i}$, where $X^{[i]}$ denotes the $i$-fold fat wedge of $X$ given by

$$
X^{[i]}=\left\{\left(x_{1}, \ldots, x_{i}\right) \in X^{i} \mid x_{j}=* \text { for some } 1 \leq j \leq i\right\} .
$$


Let $E_{i}: T_{i} \rightarrow L_{A}(X)$ be the map defined by

$$
\begin{aligned}
& \quad E_{i}\left(\delta_{k}(r, s)(\rho, \sigma), x_{1}, \ldots, x_{i}\right) \\
& \quad=F_{r}\left(\rho, x_{1}, \ldots, x_{k-1}, M_{s}\left(\sigma, x_{k}, \ldots, x_{k+s-1}\right), x_{k+s}, \ldots, x_{i}\right), \\
& \left.\quad E_{i}\left(\delta(t) r_{1}, \ldots, r_{t}\right)\left(\tau, \rho_{1}, \ldots, \rho_{t}\right), x_{1}, \ldots, x_{i}\right) \\
& \quad=N_{t}\left(\tau, F_{r_{1}}\left(\rho_{1}, x_{1}, \ldots, x_{r_{1}}\right), \ldots, F_{r_{t}}\left(x_{r_{1}+\cdots+r_{t-1}+1}, \ldots, x_{i}\right)\right)
\end{aligned}
$$

for $(k, r, s) \in \Phi_{i}$ and $\left(t ; r_{1}, \ldots, r_{t}\right) \in \Psi_{i}$,

$$
\begin{aligned}
& E_{i}\left(\gamma, x_{1}, \ldots, x_{j-1}, *, x_{j+1}, \ldots, x_{i}\right) \\
& \quad=F_{i-1}\left(d_{j}(\gamma), x_{1}, \ldots, x_{j-1}, x_{j+1}, \ldots, x_{i}\right)
\end{aligned}
$$

for $\gamma \in \Gamma_{i}$ and $1 \leq j \leq i$, and

$$
E_{i}\left(\zeta_{i}(1, \tau), x_{1}, \ldots, x_{i}\right)=\phi_{X}\left(M_{i}\left(\tau, x_{1}, \ldots, x_{i}\right)\right)
$$

for $\tau \in K_{i}$. From the homotopy extension property, there exists a map $\widetilde{E}_{i}: \Gamma_{i} \times X^{i} \rightarrow L_{A}(X)$ with $\left.\widetilde{E}_{i}\right|_{T_{i}}=E_{i}$. Let $Q_{i}: K_{i} \times X^{i} \rightarrow L_{A}(X)$ be the map given by $Q_{i}\left(\tau, x_{1}, \ldots, x_{i}\right)=\widetilde{E}_{i}\left(0, \tau, x_{1}, \ldots, x_{i}\right)$. By using a similar argument to the proof of $\left[\mathbf{1 4}\right.$, Thm. 2.14], we can construct a map $N_{i}$ : $K_{i} \times L_{A}(X)^{i} \rightarrow L_{A}(X)$ which satisfies (2.2)-(2.4) and $N_{i}\left(1_{K_{i}} \times\left(\phi_{X}\right)^{i}\right) \simeq Q_{i}$ rel $L_{i} \times X^{i}$. By using the same argument as the proof of [14, Thm. 2.14] again, we have a map $F_{i}: \Gamma_{i} \times X^{i} \rightarrow L_{A}(X)$ satisfying Conditions (2.5)-(2.8), which implies the required conclusion. This completes the proof.

In the case of the colocalization functor $C W_{A}$, we can prove Theorem 2.1(2) by using a similar argument to the proof of Theorem 2.1(1), and so we omit the proof. In the proof, we need the following result instead of $[\mathbf{1 4}$, Prop. $2.3]$ :

Proposition 2.2. Let $A, X \in \mathcal{S}_{*}$, and let $\psi_{X}: C W_{A}(X) \rightarrow X$ denote the natural map. Then we have the following homotopy equivalences:

$$
\begin{aligned}
& \left(\psi_{X}\right)_{*}: \operatorname{Map}_{*}\left(C W_{A}(X)^{i}, C W_{A}(X)\right) \longrightarrow \operatorname{Map}_{*}\left(C W_{A}(X)^{i}, X\right), \\
& \left(\psi_{X}\right)_{*}: \operatorname{Map}_{*}\left(C W_{A}(X)^{(i)}, C W_{A}(X)\right) \longrightarrow \operatorname{Map}_{*}\left(C W_{A}(X)^{(i)}, X\right)
\end{aligned}
$$

for $i \geq 1$, where $Z^{(i)}$ denotes the $i$-fold smash product of a space $Z$.

From the proof of [5, Thm. 2.E.1], we have the following lemma:

Lemma 2.3. Let $A \in \mathcal{S}_{*}$. If $f: Y \rightarrow X$ is an $A$-equivalence and $W$ is an $A$-cellular space, then the induced map

$$
f_{*}: \operatorname{Map}_{*}(W, Y) \longrightarrow \operatorname{Map}_{*}(W, X)
$$

is a homotopy equivalence. 
Proof of Proposition 2.2. By Lemma 2.3, the induced map

$$
\left(\psi_{X}\right)_{*}: \operatorname{Map}_{*}\left(W, C W_{A}(X)\right) \longrightarrow \operatorname{Map}_{*}(W, X)
$$

is a homotopy equivalence for an $A$-cellular space $W$. Then it is sufficient to show that $C W_{A}(X)^{i}$ and $C W_{A}(X)^{(i)}$ are $A$-cellular. If $B$ and $C$ are $A$-cellular, then by [5, Cor. 2.D.17], $B \times C$ is $A$-cellular. Since $B \vee C$ is represented as a homotopy colimit space, by [5, Def. 2.D.1], $B \vee C$ is $A$ cellular, and by using [5, Def. 2.D.1] again, so is $B \wedge C$. From these facts, we have the required conclusion. This completes the proof.

Let $S^{m}$ denote the $m$-dimensional sphere for $m \geq 1$. From the definition, one can see that a space $X$ is $S^{m}$-null if and only if $\pi_{i}(X)=0$ for $i \geq$ $m$, and a map $f: X \rightarrow Y$ is $S^{m}$-equivalence if and only if the induced homomorphism $f_{*}: \pi_{i}(X) \rightarrow \pi_{i}(Y)$ is an isomorphism for $i \geq m$ (see $[5,1 . A .1 .1,2 . D .2 .6])$. Furthermore, it is shown by Dror Farjoun [5, 1.E.1, 2.A.3.1] that the $S^{m}$-nullification $L_{S^{m}}(X)$ is the $(m-1)$-th stage $X_{m-1}$ of the Postnikov system of $X$, and the $S^{m}$-colocalization $C W_{S^{m}}(X)$ is the $(m-1)$-connected cover $X\langle m-1\rangle$ of $X$. Then as direct consequences of Theorem 2.1, we have the following results:

Corollary 2.4. If $X$ is an $A_{n}$-space, then the $m$-th stage $X_{m}$ of the Postnikov system is an $A_{n}$-space and the natural projection $p_{m}: X \rightarrow X_{m}$ is an $A_{n}$-map.

Corollary 2.5. If $X$ is an $A_{n}$-space, then the $m$-connected cover $X\langle m\rangle$ is an $A_{n}$-space and the natural inclusion $i_{m}: X\langle m\rangle \rightarrow X$ is an $A_{n}$-map.

Remark 2.6. By using a result of Stasheff [22, II Cor. 10.6], we have a similar result to Corollary 2.4 (see [22, II Thm. 6.2]). His result implies that we can choose $A_{n}$-forms on $X$ and $X_{m}$, so that the projection $p_{m}: X \rightarrow X_{m}$ is an $A_{n}$-homomorphism, where $A_{n}$-homomorphism is a map between $A_{n^{-}}$ spaces strictly preserving the $A_{n}$-forms (see [22, II Def. 4.1]). Corollary 2.4 has the advantage in that we need not change the given $A_{n}$-form on $X$.

\section{Proofs of Theorem A and Theorem B.}

In this section, we give the proofs of Theorem A and Theorem B. First we prove Theorem A by combining Theorem 2.1 with the results of Broto and Crespo about $H$-spaces with finitely generated cohomology. Next we show that the $B \mathbb{Z} / p$-nullification functor $L_{B \mathbb{Z} / p}$ preserves a quasi $C_{n}$-form on an $A_{n}$-space (see Theorem 3.5). By using Theorem A and Theorem 3.5, we prove Theorem B.

Lin [17] posed a question whether a simply connected $H$-space whose mod $p$ cohomology is finitely generated as an algebra has the same mod $p$ cohomology as a product of $\mathbb{C} P^{\infty} \mathrm{s}$ with three-connected covers of finite 
$H$-spaces and finite $H$-spaces (see [17, p. 1105]). Broto and Crespo [3] and [4] answered positively the question as follows:

Theorem 3.1 ([3, Thm. 1.1], [4, Thm. 1.1]). Let $p$ be a prime. If $X$ is a simply connected $\bmod p H$-space such that the $\bmod p$ cohomology $H^{*}(X ; \mathbb{Z} / p)$ is finitely generated as an algebra, then we have a simply connected mod $p$ finite $H$-space $Y$ and a principal $H$-fibration

$$
F \stackrel{\alpha}{\longrightarrow} X \stackrel{\beta}{\longrightarrow} Y,
$$

where the fiber $F$ is the direct product of a finite number of $\mathbb{C} P^{\infty}$ s.

In $[\mathbf{3}]$ and $[4]$, they also remarked the next fact without the proof (see [3, p. 354]). Since it is an essential point in the proof of Theorem A, we will explain the proof in detail.

Proposition 3.2. Suppose that $X$ satisfies the same conditions as Theorem 3.1. Then we have the following homotopy commutative diagram of fibrations:

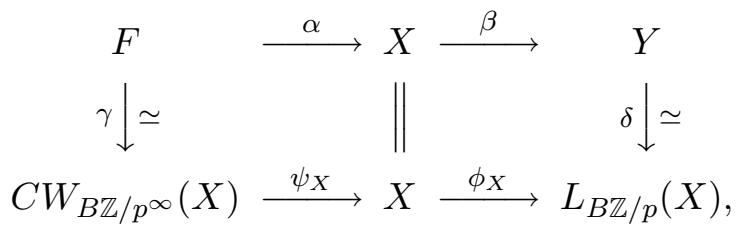

where the top horizontal sequence is the principal $H$-fibration (3.1).

Proof. By a result of Dwyer-Wilkerson [7, Thm. 9.3], we have that $\operatorname{Map}_{*}\left(B \mathbb{Z} / p^{\infty}, Y\right)$ is contractible since $Y$ is a $\bmod p$ finite $H$-space. Then $\alpha: F \rightarrow X$ is a $B \mathbb{Z} / p^{\infty}$-equivalence. By using the universality of the natural map $\psi_{X}: C W_{B \mathbb{Z} / p^{\infty}}(X) \rightarrow X$, we have a map $\xi: C W_{B \mathbb{Z} / p^{\infty}}(X) \rightarrow F$ with $\alpha \xi \simeq \psi_{X}$. Since $F$ is $B \mathbb{Z} / p^{\infty}$-cellular, by Lemma 2.3 , there exists a map $\gamma: F \rightarrow C W_{B \mathbb{Z} / p^{\infty}}(X)$ with $\psi_{X} \gamma \simeq \alpha$. By using Lemma 2.3 again, we have a bijection

$$
\left(\psi_{X}\right)_{*}:\left[C W_{B \mathbb{Z} / p^{\infty}}(X), C W_{B \mathbb{Z} / p^{\infty}}(X)\right] \longrightarrow\left[C W_{B \mathbb{Z} / p^{\infty}}(X), X\right],
$$

which implies that $\gamma \xi \simeq 1_{C W_{B \mathbb{Z} / p}(X)}$. Similarly, we have $\xi \gamma \simeq 1_{F}$ by using a bijection $\alpha_{*}:[F, F] \rightarrow[F, X]$, and thus $\gamma: F \rightarrow C W_{B \mathbb{Z} / p^{\infty}}(X)$ is a homotopy equivalence.

By Miller [19, Thm. A], $Y$ is $B \mathbb{Z} / p$-null, and so the natural map $\phi_{Y}$ : $Y \rightarrow L_{B \mathbb{Z} / p}(Y)$ is a homotopy equivalence. By a result of Dror Farjoun [5, Cor. 3.D.3], the $B \mathbb{Z} / p$-nullification functor $L_{B \mathbb{Z} / p}$ preserves the fibration (3.1). Furthermore, by [1, Remark 9.5], $L_{B \mathbb{Z} / p}(F)$ is contractible, which implies that $L_{B \mathbb{Z} / p}(\beta): L_{B \mathbb{Z} / p}(X) \rightarrow L_{B \mathbb{Z} / p}(Y)$ is a homotopy equivalence. Let $\delta=L_{B \mathbb{Z} / p}(\beta)^{-1} \phi_{Y}: Y \rightarrow L_{B \mathbb{Z} / p}(X)$, where $L_{B \mathbb{Z} / p}(\beta)^{-1}: L_{B \mathbb{Z} / p}(Y) \rightarrow$ $L_{B \mathbb{Z} / p}(X)$ denotes the homotopy inverse of $L_{B \mathbb{Z} / p}(\beta)$. Then $\delta$ is a homotopy 
equivalence with $\delta \phi_{X} \simeq \beta$, and we have the required conclusion. This completes the proof.

Now we prove Theorem A as follows:

Proof of Theorem A. Let $X$ be a simply connected $A_{n}$-space such that the $\bmod p$ cohomology $H^{*}(X ; \mathbb{Z} / p)$ is finitely generated as an algebra. By Theorem 3.1 and Proposition 3.2, we have the following principal $H$-fibration:

$$
C W_{B \mathbb{Z} / p^{\infty}}(X) \stackrel{\psi_{X}}{\longrightarrow} X \stackrel{\phi_{X}}{\longrightarrow} L_{B \mathbb{Z} / p}(X),
$$

where $C W_{B \mathbb{Z} / p^{\infty}}(X)$ is homotopy equivalent to a finite product of $\mathbb{C} P^{\infty}$ s. From Theorem 2.1, the above fibration is a principal $A_{n}$-fibration, and we have the required conclusion. This completes the proof of Theorem A.

Next we proceed to the proof of Theorem B. Hemmi $[8]$ introduced the concept of a quasi $C_{n}$-form on an $A_{n}$-space. Let $X$ be an $A_{n}$-space in the sense of Stasheff [22], and let $P_{i}(X)$ denote the $i$-th projective space of $X$ for $1 \leq i \leq n$. From the construction of $P_{i}(X)$, we have the following cofiber sequence:

$$
P_{i-1}(X) \stackrel{\iota_{i-1}}{\longrightarrow} P_{i}(X) \stackrel{\rho_{i}}{\longrightarrow}(\Sigma X)^{(i)}
$$

for $1 \leq i \leq n$. To describe the definition of a quasi $C_{n}$-form, let $\kappa_{i}$ : $(\Sigma X)^{i-1} \rightarrow(\Sigma X)^{i}$ and $\epsilon_{i}: \Sigma X \rightarrow(\Sigma X)^{i}$ be the inclusion maps given by

$$
\kappa_{i}\left(x_{1}, \ldots, x_{i-1}\right)=\left(x_{1}, \ldots, x_{i-1}, *\right)
$$

and

$$
\epsilon_{i}(x)=(*, \ldots, *, x)
$$

for $2 \leq i \leq n$. Let $\zeta_{i}:(\Sigma X)^{i} \rightarrow(\Sigma X)^{(i)}$ denote the natural projection for $1 \leq i \leq n$. A quasi $C_{n}$-form on $X$ is defined by a system of maps $\left\{\lambda_{i}:(\Sigma X)^{i} \rightarrow P_{i}(X)\right\}_{1 \leq i \leq n}$ satisfying the following conditions:

$$
\begin{array}{ll}
\lambda_{1}=1_{\Sigma X}: \Sigma X \longrightarrow \Sigma X, & \\
\lambda_{i} \kappa_{i}=\iota_{i-1} \lambda_{i-1} & \text { for } 2 \leq i \leq n, \\
\lambda_{i} \epsilon_{i}=\iota_{i-1} \cdots \iota_{1} & \text { for } 2 \leq i \leq n, \\
\rho_{i} \lambda_{i} \simeq\left(\sum_{\sigma \in \Sigma_{i}} \sigma\right) \zeta_{i} & \text { for } 1 \leq i \leq n,
\end{array}
$$

where the action of the symmetric group $\Sigma_{i}$ on $(\Sigma X)^{(i)}$ is given by the permutation of the coordinates, and the summation on the right hand side is defined by using the natural co- $H$-structure on $(\Sigma X)^{(i)}$. An $A_{n}$-space which has a quasi $C_{n}$-form is called a quasi $C_{n}$-space. Hemmi has shown the following result: 
Theorem 3.3 ([8, Thm. 1.1]). If $X$ is a simply connected finite quasi $C_{p^{-}}$ space, then $X$ is contractible.

Remark 3.4. The above definition of a quasi $C_{n}$-form is slightly weaker than the original definition due to Hemmi. In fact, he defined a quasi $C_{n^{-}}$ form by a system of maps $\left\{\psi_{i}: J_{i}(\Sigma X) \rightarrow P_{i}(X)\right\}_{1 \leq i \leq n}$ satisfying some conditions (see [8, Def. 2.1]), where $J_{i}(\Sigma X)$ denotes the $i$-th James reduced product space of $\Sigma X$ for $1 \leq i \leq n$. Given a quasi $C_{n}$-form $\left\{\psi_{i}: J_{i}(\Sigma X) \rightarrow\right.$ $\left.P_{i}(X)\right\}_{1 \leq i \leq n}$ in the sense of Hemmi, by composing $\psi_{i}$ with the natural projection $\pi_{i}:(\Sigma X)^{i} \rightarrow J_{i}(\Sigma X)$, we have a system $\left\{\lambda_{i}:(\Sigma X)^{i} \rightarrow P_{i}(X)\right\}_{1 \leq i \leq n}$ satisfying Conditions (3.2)-(3.5). Hemmi has shown Theorem 3.3 under the assumption that $X$ is a quasi $C_{p}$-space in his definition. However, one can see from his proof that our definition is also sufficient to prove Theorem 3.3.

Let $X$ and $Y$ be quasi $C_{n}$-spaces which have the quasi $C_{n}$-forms $\left\{\lambda_{i}^{X}\right.$ : $\left.(\Sigma X)^{i} \rightarrow P_{i}(X)\right\}_{1 \leq i \leq n}$ and $\left\{\lambda_{i}^{Y}:(\Sigma Y)^{i} \rightarrow P_{i}(Y)\right\}_{1 \leq i \leq n}$, respectively. If $\phi: X \rightarrow Y$ is an $A_{n}$-map, then by Iwase-Mimura [11, Thm. 3.1], we have the induced map $P_{i}(\phi): P_{i}(X) \rightarrow P_{i}(Y)$ for $1 \leq i \leq n$. Now $\phi: X \rightarrow Y$ is called a quasi $C_{n}$-map if the following diagram is homotopy commutative:

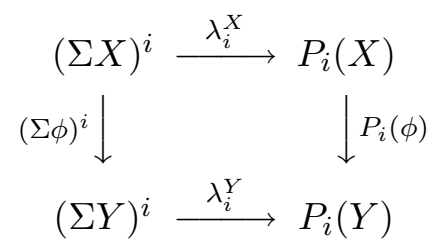

for $1 \leq i \leq n$.

In the proof of Theorem B, we need the following result:

Theorem 3.5. If $X$ is a simply connected quasi $C_{n}$-space such that the mod $p$ cohomology $H^{*}(X ; \mathbb{Z} / p)$ is finitely generated as an algebra, then $L_{B \mathbb{Z} / p}(X)$ is a quasi $C_{n}$-space and $\phi_{X}: X \rightarrow L_{B \mathbb{Z} / p}(X)$ is a quasi $C_{n}$-map.

Lemma 3.6. Suppose $X$ satisfies the same conditions as Theorem 3.5. Then we have the following:

(1) There exists a homotopy equivalence

$$
\nu_{i}:\left(\Sigma L_{B \mathbb{Z} / p}(X)\right)^{i} \rightarrow L_{B \mathbb{Z} / p}\left((\Sigma X)^{i}\right)
$$

with $\phi_{(\Sigma X)^{i}} \simeq \nu_{i}\left(\Sigma \phi_{X}\right)^{i}$ for $i \geq 1$.

(2) Given a $B \mathbb{Z} /$ p-null space $Z$, the induced map

$$
\left(\left(\Sigma \phi_{X}\right)^{i}\right)^{*}: \operatorname{Map}_{*}\left(\left(\Sigma L_{B \mathbb{Z} / p}(X)\right)^{i}, Z\right) \longrightarrow \operatorname{Map}_{*}\left((\Sigma X)^{i}, Z\right)
$$

is a homotopy equivalence for $i \geq 1$.

Proof. First we show (1). By Dror Farjoun [5, 1.A.8 e.4], there exists a homotopy equivalence $\gamma_{j}: L_{B \mathbb{Z} / p}\left((\Sigma X)^{j}\right) \times L_{B \mathbb{Z} / p}(\Sigma X) \rightarrow L_{B \mathbb{Z} / p}\left((\Sigma X)^{j+1}\right)$ such that $\gamma_{j}\left(\phi_{(\Sigma X)^{j}} \times \phi_{\Sigma X}\right) \simeq \phi_{(\Sigma X)^{j+1}}$ for $j \geq 1$. If we define a map 
$\theta_{i}: L_{B \mathbb{Z} / p}(\Sigma X)^{i} \rightarrow L_{B \mathbb{Z} / p}\left((\Sigma X)^{i}\right)$ by $\theta_{i}=\gamma_{i-1}\left(\gamma_{i-2} \times 1_{L_{B \mathbb{Z} / p}(\Sigma X)}\right) \cdots\left(\gamma_{1} \times\right.$

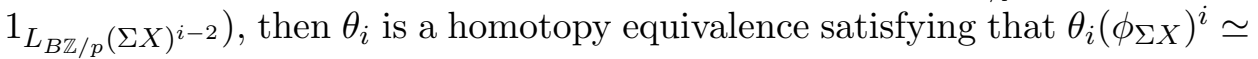
$\phi_{(\Sigma X)^{i}}$ for $i \geq 1$.

From [14, Lemma 2.6], we have a homotopy equivalence $\omega: L_{B \mathbb{Z} / p}(\Sigma X) \rightarrow$ $L_{B \mathbb{Z} / p}\left(L_{B \mathbb{Z} / p}\left(S^{1}\right) \wedge L_{B \mathbb{Z} / p}(X)\right)$ such that

$$
\omega \phi_{\Sigma X} \simeq \phi_{L_{B \mathbb{Z} / p}\left(S^{1}\right) \wedge L_{B \mathbb{Z} / p}(X)}\left(\phi_{S^{1}} \wedge \phi_{X}\right) .
$$

Let $\omega^{-1}: L_{B \mathbb{Z} / p}\left(L_{B \mathbb{Z} / p}\left(S^{1}\right) \wedge L_{B \mathbb{Z} / p}(X)\right) \rightarrow L_{B \mathbb{Z} / p}(\Sigma X)$ denote the homotopy inverse of $\omega$. By Theorem $\mathrm{A}, L_{B \mathbb{Z} / p}(X) \simeq Y$ is finite, and so is $L_{B \mathbb{Z} / p}\left(S^{1}\right) \wedge L_{B \mathbb{Z} / p}(X) \simeq \Sigma L_{B \mathbb{Z} / p}(X)$. Then $\phi_{L_{B \mathbb{Z} / p}\left(S^{1}\right) \wedge L_{B \mathbb{Z} / p}(X)}$ is a homotopy equivalence. Let $\zeta: \Sigma L_{B \mathbb{Z} / p}(X) \rightarrow L_{B \mathbb{Z} / p}(\Sigma X)$ be the map defined by $\zeta=\omega^{-1} \phi_{L_{B \mathbb{Z} / p}\left(S^{1}\right) \wedge L_{B \mathbb{Z} / p}(X)}\left(\phi_{S^{1}} \wedge 1_{L_{B \mathbb{Z} / p}(X)}\right)$. Then $\zeta$ is a homotopy equivalence and $\zeta\left(\Sigma \phi_{X}\right) \simeq \phi_{\Sigma X}$. If we put $\nu_{i}=\theta_{i} \zeta^{i}:\left(\Sigma L_{B \mathbb{Z} / p}(X)\right)^{i} \rightarrow$ $L_{B \mathbb{Z} / p}\left((\Sigma X)^{i}\right)$, then $\nu_{i}$ satisfies the required conditions.

Next we show (2). By taking the mapping spaces, we have the following homotopy commutative diagram:

$$
\begin{array}{ccc}
\operatorname{Map}_{*}\left(L_{B \mathbb{Z} / p}\left((\Sigma X)^{i}\right), Z\right) \stackrel{\nu_{i}^{*}}{\longrightarrow} \operatorname{Map}_{*}\left(\left(\Sigma L_{B \mathbb{Z} / p}(X)\right)^{i}, Z\right) \\
\left(\phi_{(\Sigma X)^{i}}\right)^{*} \downarrow \simeq & \downarrow\left(\left(\Sigma \phi_{X}\right)^{i}\right)^{*} \\
\operatorname{Map}_{*}\left((\Sigma X)^{i}, Z\right) & = & \\
\operatorname{Map}_{*}\left((\Sigma X)^{i}, Z\right)
\end{array}
$$

for a $B \mathbb{Z} / p$-null space $Z$. Since $\nu_{i}^{*}$ and $\left(\phi_{(\Sigma X)^{i}}\right)^{*}$ are homotopy equivalences by (1) and (2.1), so is $\left(\left(\Sigma \phi_{X}\right)^{i}\right)^{*}$. This completes the proof.

Proof of Theorem 3.5. Let $X$ be an $A_{n}$-space with finitely generated mod $p$ cohomology. From Theorem 2.1, the $B \mathbb{Z} / p$-nullification $L_{B \mathbb{Z} / p}(X)$ is an $A_{n}$-space and the natural map $\phi_{X}: X \rightarrow L_{B \mathbb{Z} / p}(X)$ is an $A_{n}$-map. Then by Iwase-Mimura [11, Thm. 3.1], we have the induced map $P_{i}\left(\phi_{X}\right): P_{i}(X) \rightarrow$ $P_{i}\left(L_{B \mathbb{Z} / p}(X)\right)$ for $1 \leq i \leq n$ satisfying the following homotopy commutative diagram of cofiber sequences:

$$
\begin{aligned}
& P_{i-1}(X) \stackrel{\iota_{i-1}}{\longrightarrow} P_{i}(X) \stackrel{\rho_{i}}{\longrightarrow} \quad(\Sigma X)^{(i)} \\
& P_{i-1}\left(\phi_{X}\right) \downarrow \quad P_{i}\left(\phi_{X}\right) \downarrow \quad \downarrow\left(\Sigma \phi_{X}\right)^{(i)} \\
& P_{i-1}\left(L_{B \mathbb{Z} / p}(X)\right) \stackrel{\widetilde{\iota}_{i-1}}{\longrightarrow} P_{i}\left(L_{B \mathbb{Z} / p}(X)\right) \stackrel{\widetilde{\rho}_{i}}{\longrightarrow}\left(\Sigma L_{B \mathbb{Z} / p}(X)\right)^{(i)},
\end{aligned}
$$

where $\widetilde{\iota}_{i-1}: P_{i-1}\left(L_{B \mathbb{Z} / p}(X)\right) \rightarrow P_{i}\left(L_{B \mathbb{Z} / p}(X)\right)$ is the natural inclusion and $\tilde{\rho}_{i}: P_{i}\left(L_{B \mathbb{Z} / p}(X)\right) \rightarrow\left(\Sigma L_{B \mathbb{Z} / p}(X)\right)^{(i)}$ denotes the natural projection. Since $L_{B \mathbb{Z} / p}(X)$ is finite, so is $\left(\Sigma L_{B \mathbb{Z} / p}(X)\right)^{(i)}$ for $i \geq 1$. By using induction on $i$, the projective space $P_{i}\left(L_{B \mathbb{Z} / p}(X)\right)$ is finite, and so $P_{i}\left(L_{B \mathbb{Z} / p}(X)\right)$ is $B \mathbb{Z} / p$-null. Then there exists a map $\eta_{i}: L_{B \mathbb{Z} / p}\left(P_{i}(X)\right) \rightarrow P_{i}\left(L_{B \mathbb{Z} / p}(X)\right)$ 
such that $\eta_{i} \phi_{P_{i}(X)} \simeq P_{i}\left(\phi_{X}\right)$. Now we define a map $\hat{\lambda}_{i}:\left(\Sigma L_{B \mathbb{Z} / p}(X)\right)^{i} \rightarrow$ $P_{i}\left(L_{B \mathbb{Z} / p}(X)\right)$ by the composite $\hat{\lambda}_{i}=\eta_{i} L_{B \mathbb{Z} / p}\left(\lambda_{i}\right) \nu_{i}$. Then by Lemma 3.6, we see that $\hat{\lambda}_{i}\left(\Sigma \phi_{X}\right)^{i} \simeq P_{i}\left(\phi_{X}\right) \lambda_{i}$ for $1 \leq i \leq n$. If we show that the system $\left\{\hat{\lambda}_{i}:\left(\Sigma L_{B \mathbb{Z} / p}(X)\right)^{i} \rightarrow P_{i}\left(L_{B \mathbb{Z} / p}(X)\right)\right\}_{1 \leq i \leq n}$ is a quasi $C_{n}$-form on $L_{B \mathbb{Z} / p}(X)$, then the result follows.

First we consider Conditions (3.3) and (3.4). Let $\widetilde{\kappa}_{i}:\left(\Sigma L_{B \mathbb{Z} / p}(X)\right)^{i-1}$ $\rightarrow\left(\Sigma L_{B \mathbb{Z} / p}(X)\right)^{i}$ be the inclusion given by

$$
\widetilde{\kappa}_{i}\left(y_{1}, \ldots, y_{i-1}\right)=\left(y_{1}, \ldots, y_{i-1}, *\right)
$$

for $2 \leq i \leq n$. From the definition of $\hat{\lambda}_{i}$, and by using Diagram (3.6), we have that $\left(\left(\Sigma \phi_{X}\right)^{i-1}\right)^{*}\left(\left[\hat{\lambda}_{i} \widetilde{\kappa}_{i}\right]\right)=\left[P_{i}\left(\phi_{X}\right) \lambda_{i} \kappa_{i}\right]$ and $\left(\left(\Sigma \phi_{X}\right)^{i-1}\right)^{*}\left(\left[\widetilde{\iota}_{i-1} \hat{\lambda}_{i-1}\right]\right)=$ $\left[P_{i}\left(\phi_{X}\right) \iota_{i-1} \lambda_{i-1}\right]$. Then $\left(\left(\Sigma \phi_{X}\right)^{i-1}\right)^{*}\left(\left[\hat{\lambda}_{i} \widetilde{\kappa}_{i}\right]\right)=\left(\left(\Sigma \phi_{X}\right)^{i-1}\right)^{*}\left(\left[\widetilde{\iota}_{i-1} \hat{\lambda}_{i-1}\right]\right)$ since $\lambda_{i} \kappa_{i}=\iota_{i-1} \lambda_{i-1}$. By Lemma 3.6,

$$
\begin{aligned}
\left(\left(\Sigma \phi_{X}\right)^{i-1}\right)^{*}:\left[\left(\Sigma L_{B \mathbb{Z} / p}(X)\right)^{i-1}, P_{i-1}\left(L_{B \mathbb{Z} / p}(X)\right)\right] & \\
& \longrightarrow\left[(\Sigma X)^{i-1}, P_{i-1}\left(L_{B \mathbb{Z} / p}(X)\right)\right]
\end{aligned}
$$

is a bijection since $P_{i-1}\left(L_{B \mathbb{Z} / p}(X)\right)$ is $B \mathbb{Z} / p$-null, which implies that $\hat{\lambda}_{i} \widetilde{\kappa}_{i} \simeq$ $\widetilde{\iota}_{i-1} \hat{\lambda}_{i-1}$ for $2 \leq i \leq n$. By using the same argument as above, we have $\hat{\lambda}_{i} \widetilde{\epsilon}_{i} \simeq \widetilde{\iota}_{i-1} \cdots \widetilde{\iota}_{1}$ for $2 \leq i \leq n$, where $\widetilde{\epsilon}_{i}: \Sigma L_{B \mathbb{Z} / p}(X) \rightarrow\left(\Sigma L_{B \mathbb{Z} / p}(X)\right)^{i}$ denotes the inclusion given by

$$
\widetilde{\epsilon}_{i}(y)=(*, \ldots, *, y)
$$

for $2 \leq i \leq n$. From the homotopy extension property, we can choose a map $\widetilde{\lambda}_{i}:\left(\Sigma L_{B \mathbb{Z} / p}(X)\right)^{i} \rightarrow P_{i}\left(L_{B \mathbb{Z} / p}(X)\right)$ such that $\widetilde{\lambda}_{i} \simeq \hat{\lambda}_{i}$ for $1 \leq i \leq n$ and the system $\left\{\widetilde{\lambda}_{i}\right\}_{1 \leq i \leq n}$ satisfies Conditions (3.3) and (3.4).

Next we show Condition (3.5). Let $\widetilde{\zeta}_{i}:\left(\Sigma L_{B \mathbb{Z} / p}(X)\right)^{i} \rightarrow\left(\Sigma L_{B \mathbb{Z} / p}(X)\right)^{(i)}$ denote the natural projection for $1 \leq i \leq n$. Since $X$ is a quasi $C_{n}$-space, by using Condition (3.5) and the homotopy commutativity of Diagram (3.6), we have that

$$
\left(\left(\Sigma \phi_{X}\right)^{i}\right)^{*}\left(\left[\widetilde{\rho}_{i} \widetilde{\lambda}_{i}\right]\right)=\left[\left(\Sigma \phi_{X}\right)^{(i)} \rho_{i} \lambda_{i}\right]=\left[\left(\Sigma \phi_{X}\right)^{(i)}\left(\sum_{\sigma \in \Sigma_{i}} \sigma\right) \zeta_{i}\right] .
$$

On the other hand, we see that

$$
\left(\left(\Sigma \phi_{X}\right)^{i}\right)^{*}\left(\left[\left(\sum_{\sigma \in \Sigma_{i}} \sigma\right) \widetilde{\zeta}_{i}\right]\right)=\left[\left(\Sigma \phi_{X}\right)^{(i)}\left(\sum_{\sigma \in \Sigma_{i}} \sigma\right) \zeta_{i}\right]
$$


since $\left(\Sigma \phi_{X}\right)^{(i)}:(\Sigma X)^{(i)} \rightarrow\left(\Sigma L_{B \mathbb{Z} / p}(X)\right)^{(i)}$ is a co- $H$-map. By applying Lemma 3.6 to a $B \mathbb{Z} / p$-null space $\left(\Sigma L_{B \mathbb{Z} / p}(X)\right)^{(i)}$, we have a bijection

$$
\left(\left(\Sigma \phi_{X}\right)^{i}\right)^{*}:\left[\left(\Sigma L_{B \mathbb{Z} / p}(X)\right)^{i},\left(\Sigma L_{B \mathbb{Z} / p}(X)\right)^{(i)}\right] \longrightarrow\left[(\Sigma X)^{i},\left(\Sigma L_{B \mathbb{Z} / p}(X)\right)^{(i)}\right],
$$

which implies that

$$
\widetilde{\rho}_{i} \widetilde{\lambda}_{i} \simeq\left(\sum_{\sigma \in \Sigma_{i}} \sigma\right) \widetilde{\zeta}_{i}
$$

for $1 \leq i \leq n$. This completes the proof of Theorem 3.5.

Now we can prove Theorem B as follows:

Proof of Theorem B. Let $X$ be a simply connected quasi $C_{p}$-space such that the $\bmod p$ cohomology $H^{*}(X ; \mathbb{Z} / p)$ is finitely generated as an algebra. From Theorem $\mathrm{A}$, we have a principal $A_{p}$-fibration

$$
F \longrightarrow X \stackrel{\phi_{X}}{\longrightarrow} L_{B \mathbb{Z} / p}(X),
$$

where the fiber $F$ is homotopy equivalent to the direct product of a finite number of $\mathbb{C} P^{\infty}$ s. By Theorem 3.5, the $B \mathbb{Z} / p$-nullification $L_{B \mathbb{Z} / p}(X)$ is a simply connected finite quasi $C_{p}$-space, and by Theorem $3.3, L_{B \mathbb{Z} / p}(X)$ is contractible. Hence $X$ is homotopy equivalent to the fiber $F$, and we have the required conclusion. This completes the proof of Theorem B.

\section{References}

[1] J. Aguadé, C. Broto and D. Notbohm, Homotopy classification of spaces with interesting cohomology and a conjecture of Cooke, Part I, Topology, 33 (1994), 455-492, MR 95i:55006, Zbl 0843.55007.

[2] A. Bousfield and D. Kan, Homotopy limits, completions and localizations, Springer Lecture Notes in Math., 304 (1972), MR 51 \#1825, Zbl 0259.55004.

[3] C. Broto and J.A. Crespo, H-spaces with noetherian mod two cohomology algebra, Topology, 38 (1999), 353-386, MR 99i:55013, Zbl 0927.55017.

[4] J.A. Crespo, Structure of mod $p H$-spaces with finiteness conditions, Progr. Math., 196, Birkhäuser, Basel, 2001, CMP 1851251.

[5] E. Dror Farjoun, Cellular spaces, null spaces and homotopy localization, Springer Lecture Notes in Math., 1622 (1996), MR 98f:55010, Zbl 0842.55001.

[6] W.G. Dwyer and C.W. Wilkerson, Homotopy fixed point methods for Lie groups and finite loop spaces, Ann. of Math., 139 (1994), 395-442, MR 95e:55019, Zbl 0801.55007.

[7] _ The center of a p-compact group, Contemp. Math., 181 (1995), 119-157, MR 96a:55024, Zbl 0828.55009.

[8] Y. Hemmi, Higher homotopy commutativity of $H$-spaces and the $\bmod p$ torus theorem, Pacific J. Math., 149 (1991), 95-111, MR 92a:55010, Zbl 0691.55007. 
[9] K. Iriye and A. Kono, Mod p retracts of G-product spaces, Math. Z., 190 (1985), 357-363, MR 88a:55017, Zbl 0554.55004.

[10] N. Iwase, A continuous localization and completion, Trans. Amer. Math. Soc., 320 (1990), 77-90, MR 90k:55015, Zbl 0698.55007.

[11] N. Iwase and M. Mimura, Higher homotopy associativity, Springer Lecture Notes in Math., 1370 (1986), 193-220, MR 90f:55017, Zbl 0677.55010.

[12] R.M. Kane, The homology of Hopf spaces, North-Holland Math. Library, 40 (1988), MR 90f:55018, Zbl 0651.55001.

[13] Y. Kawamoto, Loop spaces of H-spaces with finitely generated cohomology, Pacific J. Math., 190 (1999), 311-328, MR 2000i:55027.

[14] _ Homotopy classification of higher homotopy commutative loop spaces with finitely generated cohomology, Hiroshima Math. J., 30 (2000), 317-344, MR 2001e:55011, Zbl 0958.55010.

[15] Y. Kawamoto and J.P. Lin, Homotopy commutativity of $H$-spaces with finitely generated cohomology, Trans. Amer. Math. Soc., 353(11) (2001), 4481-4496 (electronic), CMP 1851180.

[16] J.P. Lin, Loops of $H$-spaces with finitely generated cohomology rings, Topology Appl., 60 (1994), 131-152, MR 95m:55022, Zbl 0837.55012.

[17] _ H H-spaces with finiteness conditions, Handbook of Algebraic Topology, edited by I.M. James, North-Holland (1995), 1095-1141, MR 97c:55017, Zbl 0941.55002.

[18] C. McGibbon, Homotopy commutativity in localized groups, Amer. J. Math., 106 (1984), 665-687, MR 86a:55011, Zbl 0574.55004.

[19] H.R. Miller, The Sullivan conjecture on maps from classifying spaces, Ann. of Math., 120 (1984), 39-87, MR 87k:55020, Zbl 0552.55014.

[20] L. Saumell, Higher homotopy commutativity in localized groups, Math. Z., 219 (1995), 203-213, MR 96g:57044, Zbl 0821.55009.

[21] M. Slack, A classification theorem for homotopy commutative mod $2 H$-spaces with finitely generated cohomology rings, Mem. Amer. Math. Soc., 92 (1991), MR 92k:55015, Zbl 0755.55003.

[22] J.D. Stasheff, Homotopy associativity of H-spaces I, II, Trans. Amer. Math. Soc., 108 (1963), 275-292, 293-312, MR 28 \#1623, Zbl 0114.39402.

[23] _ H-spaces from a homotopy point of view, Springer Lecture Notes in Math., 161 (1970), MR 42 \#5261, Zbl 0205.27701.

[24] M. Sugawara, On the homotopy commutativity of groups and loop spaces, Mem. College Sci. Univ. Kyoto Ser. A, 33 (1960), 257-269, MR 22 \#11394, Zbl 0113.16903.

[25] F.D. Williams, Higher homotopy-commutativity, Trans. Amer. Math. Soc., 139 (1969), 191-206, MR 39 \#2163, Zbl 0185.27103.

Received July 14, 2000 and revised July 3, 2001.

Department of Mathematics

National Defense Academy

1-10-20, HASHIRIMIZU

YOKOSUKA 239-8686, JAPAN

E-mail address: yusuke@cc.nda.ac.jp 